\title{
Collaborative filtering via sparse Markov random fields
}

\author{
Truyen Tran*, Dinh Phung, Svetha Venkatesh \\ Center for Pattern Recognition and Data Analytics, Deakin University, Geelong, Victoria, Australia \\ ${ }^{*}$ Corresponding author. E-mail address: truyen.tran@deakin.edu.au
}

\begin{abstract}
Recommender systems play a central role in providing individualized access to information and services. This paper focuses on collaborative filtering, an approach that exploits the shared structure among mind-liked users and similar items. In particular, we focus on a formal probabilistic framework known as Markov random fields (MRF). We address the open problem of structure learning and introduce a sparsity-inducing algorithm to automatically estimate the interaction structures between users and between items. Item-item and user-user correlation networks are obtained as a by-product. Large-scale experiments on movie recommendation and date matching datasets demonstrate the power of the proposed method.
\end{abstract}

Keywords: Recommender systems, collaborative filtering, Markov random field, sparse graph learning, movie recommendation, dating recommendation

\section{Introduction}

Learning to recommend is powerful. It offers targeted access to information and services without requiring users to formulate explicit queries. As the recommender system observes the users, it gradually acquires users tastes and preferences to make recommendation. Yet its recommendation can be accurate and sometimes surprising. Recommender systems are now pervasive at every corner of digital life, offering diverse recommendations from books [13], learning courses [6], TV programs [2], news [4], and many others (see [16] for an up-to-date survey on applications).

An important direction to recommendation is collaborative filtering $(\mathrm{CF})$. $\mathrm{CF}$ is based on the premise that people are interested in common items, and thus there exists a shared structure that enables transferring one's preference to like-minded users. A highly interpretable approach is correlation-based, in that our future preference will be predicted based on either similar users who share the rating history [19], or correlated items that share the previous raters [22]. For example, the popular line "people who 
buy this [book] also buy ..." is likely to reflect the correlated items method. While this is intuitive, simple correlation methods might not be effective for several reasons. First, correlation is heuristic and there is little formal theory that links correlation to recommendation performance. Second, combining user and item correlations is desirable but not straightforward. Third, a recommendation should be equipped with a confidence score, but this is lacking in correlation-based methods.

A principled method that addresses these three issues is Preference Network [25]. A Preference Network is a Markov random field whose nodes represent preferences by a user on an item, and edges represent the dependency between items and between users. The shared structure is encapsulated in the model parameters and enables future prediction. Model parameters that measure association strength between items and between users are jointly estimated to maximize the agreement with the data and model prediction. Prediction is based on the most probable assignment, which comes with quantifiable confidence.

More recent variants and extensions of Preference Network have been subsequently introduced [5, 7, 15, 14, 28]. However, one important problem still remains, that is the to estimate the model structure automatically from data. Previous work was mainly based on heuristics that pick an edge if the correlation is beyond a predefined threshold. To that end, we propose a sparsity-inducing framework to learn the edges of the Markov random field directly from data while maximizing the agreement between data and prediction. It results in a sparse network, where each item (or user) is connected to only a handful of other items (or users). Thus it is optimal with respect to the prediction task, and it frees the model designer from specifying the structure and justifying the choice. With tens of thousands of users and items, our MRFs - with hundreds of millions of free parameters - are among the largest MRFs ever studied. With such a scale, we show how learning is possible using ordinary computers.

We study the capacity of the proposed framework on two online applications: movie recommendation and match making. In movie recommendation, users provide ratings for each movie they have watched, and the task is to predict rating for unseen movies. Likewise in match making, each user rates a number of profiles of other users, and the recommendation is to predict how much the user likes new profiles. The movie dataset is MovieLens $1 \mathrm{M}$ with 1 million ratings by nearly 6 thousand users on approximately 4 thousand movies. The match making dataset is Dating Agency with 17 million ratings by 135 thousand users over 169 thousand profiles. We show that the MRF-based framework outperforms well-studied baselines in various scenarios.

To summary, our main contribution is a framework for learning structures of Markov random 
fields for collaborative filtering. A by product of our structure learning framework are item and user correlation networks, which are useful for further analysis. This extends our previous work on Preference Network [25], both in theory and applications (using new datasets with several orders of magnitude larger). The rest of the paper is organized as follows. Sec.2 2 reviews related work. Sec. 3 presents our contributions in parameterizations and structure learning. The proposed frameworks are evaluated extensively in Sec. 4 . Sec. 5 concludes the paper.

\section{Background}

This section reviews existing work in collaborative filtering (CF) in general, and presents a detailed account on Markov random fields for CF in particular.

\subsection{Collaborative filtering}

Recommender systems offer fast personalized access to products and services and have found applications in numerous places [16, 18, 23]. The collaborative filtering approach to recommender systems is based on the idea that personal preferences can be collaboratively determined by mind-liked users. In a typical explicit setting, a recommender system maintains a rating database by a set of existing users on a set of available items. The database can be represented as a sparse rating matrix, where typically only less than few percents of the cells are filled. New recommendations will be made for each user for unseen items without the need of issuing an explicit query. The most common task is to predict the rating for unseen items, or equivalently, filling the empty cells in the rating matrix. For that reason, the task is sometimes referred to as matrix completion. In implicit settings, preferences are not given (e.g., clicks and music streaming). In this paper, we focus mainly on explicit settings.

The earliest and still most popular methods are dependency-based, i.e., co-rated items are correlated and co-raters are interdependent. The usual technique is $k$-nearest neighbors tailored to collaborative filtering, which can be user-based or item-based. The user-based method posits that a preference can be borrowed from like-minded users [19]. For example, the rating $r_{u i}$ by user $u$ on item $i$ is predicted as

$$
r_{u i}=\bar{r}_{u}+\frac{\sum_{v \in U(i)} s(u, v)\left(r_{v i}-\bar{r}_{v}\right)}{\sum_{v \in U(i)}|s(u, v)|}
$$

where $s(u, v)$ is the correlation between user $u$ and user $v, U(i)$ is the set of all users who rated item $i$, and $\bar{r}_{u}$ is the average rating by user $u$. Note that $s(u, v)$ can be negative, i.e., two users have opposite 


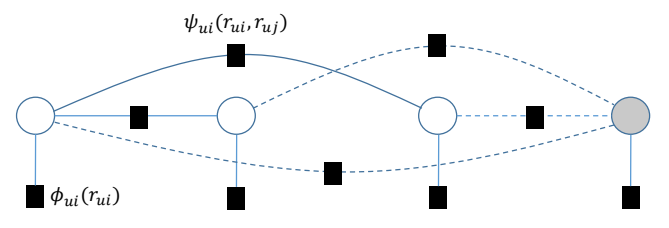

Figure 1: Markov random field for a user, represented as a factor graph. Round nodes represent ratings, filled squares represent potentials (also known as factors), and edges represent pairwise interaction. New rating is represented as a shaded node. Dashed lines indicate new edges that are added at test time and borrowed from other users.

tastes. The item-based method predicts rating for a new item based on ratings of other similar items that the user has rated [22]. This is identical to the user-based method but with the roles of user and item swapped. The two similarity methods suggest a hybrid that fuses the two predictions [27]. The computation of the similarity is critical to the success of the approach. The most common measure is Pearson's correlation. The main drawback of nearest neighbor is lack of theoretical justification of the choice of similarity measures and the computation of rating.

A more formal method is dependency networks [9] which provide a probabilistic interpretation. However, dependency networks do not offer a consistent probability measure across predictions, thus limiting its fusion capacity. Markov random fields (MRFs) eliminate this problem. The first MRF-based recommender system was introduced in [25] on which the present paper is extended. Factor-graphs, as an alternative representation of MRF [28], have been introduced for collaborative filtering but no learning was done. Rather, the MRF is merely a smoothing mechanism. Learnt MRFs were investigated in [5, 7] but these are an user-specific version of [25]. More recently, [15] extends [25] to incorporate matrix factorization, but it is still limited to user-specific MRFs.

Latent aspects represent another major approach to collaborative filtering. Examples include matrix factorization [20], RBM [21, 26], PLSA [11] and LDA [17]. These methods assume a low dimensional representation of rating data, which, once learnt, can be used to generate unseen ratings. There are evidences suggesting that the dependency-based and latent aspects approaches are complementary [26, 12]. 


\subsection{Markov random fields}

Markov random field (MRF) is a graph connecting random variables. A graph is defined as $G=(V, E)$, where $V$ is the set of nodes and $E$ is the set of edges (see Fig. 11). Each node represents a random variable. For our purpose, the random variables $x$ are discrete, and we assume that their values are drawn from a set $\{1,2, \ldots, K\}$. The contribution of each variable is encoded in a positive function called singleton potential (or factor) $\phi_{i}\left(x_{i}\right)$. An edge connecting two variables specifies a direct association between the two variables, and the association strength is quantified by a nonnegative pairwise potential $\phi_{i j}\left(x_{i}, x_{j}\right)$ for variable pair $\left(x_{i}, x_{j}\right)$. Note that the absence of an edge does not rule out the higher-order dependency between two variables.

The joint distribution for all variables is then defined as:

$$
P(x)=\frac{1}{Z} \exp (-E(x))
$$

where $Z$ is the normalizing constant to ensure that $\sum_{x} P(x)=1$ and $E(x)$ is model energy, defined as

$$
E(x)=-\left(\sum_{i \in V} \log \phi_{i}\left(x_{i}\right)+\sum_{(i, j) \in E} \log \psi_{i j}\left(x_{i}, x_{j}\right)\right)
$$

A low energy implies high probability, which translates to high compatibility between variable assignments.

The Hammersley-Clifford theorem [8] asserts that, given variable assignments of one's neighborhood, the local probability is independent of all other variables:

$$
P\left(x_{i} \mid x_{\neg i}\right)=P\left(x_{i} \mid x_{N(i)}\right) \propto \exp \left(-E\left(x_{i}, x_{N(i)}\right)\right)
$$

where $x_{\neg i}$ denotes all variables except for $x_{i}, N(i)$ is the set of nodes connected to $i$, and

$$
E\left(x_{i}, x_{N(i)}\right)=-\left(\log \phi_{i}\left(x_{i}\right)+\sum_{j \in N(i)} \log \psi_{i j}\left(x_{i}, x_{j}\right)\right)
$$

The neighborhood $N(i)$ is also known as the Markov blanket. This theorem is important because $P\left(x_{i} \mid x_{N(i)}\right)$ costs only $K$ time to compute, whereas $P(x)$ cannot be evaluated in polynomial time. Many approximate computations will rely on this property.

\subsection{MRF for a user (or an item)}

Recall that in the neighborhood-based approach, the correlation between users (or items) must be estimated, e.g., the $s(u, v)$ in Eq. (1). Ideally the estimation for all user pairs should be directly related 
to the final performance measure. A second requirement is that when making a prediction, we should be able to quantify the confidence of the prediction. Third, there should be an effective way to combine user-based and item-based methods. Markov random fields offer a principled method to meet all three criteria.

Let us start with a MRF per user [25] and then move to joint MRF for all users in Sec. 2.4. Here the ratings $r=\left(r_{1}, r_{2}, \ldots\right)$ by the user play the role of random variables. The key is to observe that items rated by the same user tend to correlate as they reflect user's tastes. Thus each user is represented by a graph $G=(V, E)$, where $V$ is the set of items rated by the user and $E$ is the set of edges connecting those related items. Each node in $V$ represents a rating variable $r_{u i}$. A graphical illustration of the MRF is given in Fig. 1 .

Let $\phi_{u i}\left(r_{u i}\right)$ be potential function that measures the compatibility of the rating $r_{u i}$ with user $u$ and item $i$, and $\psi_{i j}\left(r_{u i}, r_{u j}\right)$ encodes the pairwise relationship between two items $(i, j)$. The model energy is:

$$
E(r)=-\left(\sum_{i \in V} \log \phi_{u i}\left(r_{u i}\right)+\sum_{(i, j) \in E} \log \psi_{i j}\left(r_{u i}, r_{u j}\right)\right)
$$

A low energy signifies a high compatibility between item-user, and between item-item. The local predictive distribution, following Eq. (2), is:

$$
P\left(r_{u i} \mid r_{\neg u i}\right) \propto \exp \left(-E\left(r_{u i}, r_{N(i)}\right)\right)
$$

where

$$
E\left(r_{u i}, r_{N(i)}\right)=-\left(\log \phi_{u i}\left(r_{u i}\right)+\sum_{j \in N(i)} \log \psi_{i j}\left(r_{u i}, r_{u j}\right)\right)
$$

As each user only rates a handful of items, it is more efficient to model only the items each user has rated. Thus the MRFs for all users will be of different sizes and incorporate different item sets. For the entire system to make sense, all MRFs must relate in some way. The key here is that all user-specific models share the same set of parameters. This parameter sharing enables prediction for unseen ratings, as we present hereafter.

\subsubsection{Rate prediction}

A fully-specified MRF enables rate prediction of unseen item for reach user $u$. It is natural to suggest that the best rating will be the most probable among all ratings, conditioned on existing ratings, 
i.e.:

$$
r_{u j}^{*}=\arg \max _{r_{u j}} P\left(r_{u j} \mid r\right)=\arg \max _{r_{u j}} P\left(r_{u j} \mid r_{N(j)}\right)=\arg \min _{r_{u j}} E\left(r_{u j}, r_{N(j)}\right)
$$

where $N(j)$ is the set of seen items that are connected to $j$ and $E\left(r_{u j}, r_{N(j)}\right)$ is the local energy computed as in Eq. (5). This is a direct application of the Hammersley-Clifford theorem. A MRF not only can predict new rating $r_{u j}^{*}$, it also provides the confidence in the prediction through $P\left(r_{u j}^{*} \mid r_{N(j)}\right)$. This property also enables predicting an expected rating:

$$
\bar{r}_{u j}=\sum_{k=1}^{K} P\left(r_{u j}=k \mid r_{N(j)}\right) k
$$

where all probabilities are taken into account.

\subsubsection{Learning}

Learning is to estimate parameters of the potentials $\phi_{u i}\left(r_{u i}\right)$ and $\psi_{i j}\left(r_{u i}, r_{u j}\right)$. We aim to minimize the disagreement between the data and the model prediction, typically through the likelihood function $P(r)$. However, estimating the likelihood is generally intractable due to the exponentially large space of all possible rating assignments. In our previous work [25], the negative log pseudo-likelihood [3] loss was minimized:

$$
\mathscr{L}_{P L}=-\sum_{i \in V} \log P\left(r_{u i} \mid r_{\neg i}\right)
$$

where $P\left(r_{u i} \mid r_{\neg u i}\right)$ is defined in Eq. (4). While this loss function is only an approximation to the full negative log-likelihood, it is appealing because it has the same functional form as the predictive distribution used in rating prediction of Eq. (6).

\subsubsection{MRF for an item}

Analogous to the case of building a MRF for a user, we can also build a MRF for an item. This is because one can rotate the rating matrix and swap the roles of users and items. Under this view, users are now naturally dependent under item-based models. In particular, the model energy in Eq. (3) can be rewritten for each item $i$ as

$$
E(r)=-\left(\sum_{u \in V} \log \phi_{u i}\left(r_{u i}\right)+\sum_{(u, v) \in E} \log \varphi_{u v}\left(r_{u i}, r_{v i}\right)\right)
$$

This poses a question to integrate the user-based and item-based views, which we present next. 


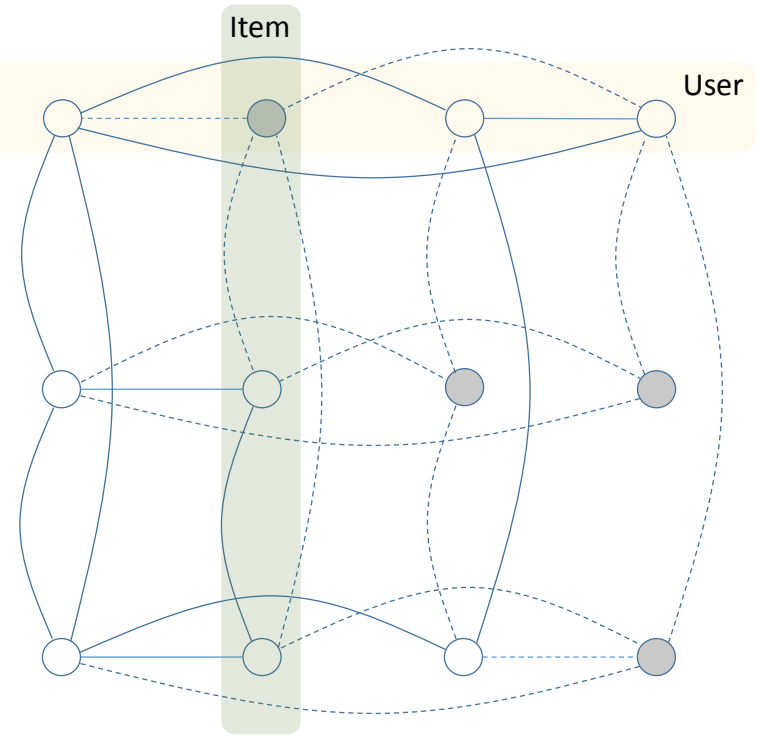

Figure 2: Markov random field for full rating database modeling. Factor nodes in Fig. 1 are dropped for clarity. When new ratings are predicted (as shade nodes), their edges are borrowed from other users and items. Note that we predict one rating at a time, so there is no direct edges between unseen ratings. 


\subsection{MRF for entire rating database}

The user-specific and item-specific models presented in the previous subsections are built upon an unstated assumption that users or items are drawn randomly and independently from the population. However, this assumption is unrealistic. First the set of items that an user rates is subject to availability at the time of rating, and the availability is evident for all other users. Second, users can be influenced by other users either implicitly (e.g., an item gets noticed due to its popularity) or explicitly (e.g., through social contacts). Thus users' choices are not entirely independent.

We build a single large MRF by joining all user-based and item-based MRFs together, as illustrated in Fig. 2. Denote by $\mathscr{R}$ the entire rating database, the full joint model energy is:

$$
E(\mathscr{R})=-\left(\sum_{r_{i j} \in \mathscr{R}} \log \phi_{u i}\left(r_{u i}\right)+\sum_{u} \sum_{(i, j) \in E(u)} \log \psi_{i j}\left(r_{u i}, r_{u j}\right)+\sum_{i} \sum_{(u, v) \in E(i)} \log \varphi_{u v}\left(r_{u i}, r_{v i}\right)\right)
$$

where $E(u)$ and $E(i)$ are the set of edges specific to user $u$ and item $i$, respectively. Applying the Hammersley-Clifford theorem in Eq. (2), the local predictive distribution becomes:

$$
P\left(r_{u i} \mid \mathscr{R}_{\neg u i}\right) \propto \exp \left(-E\left(r_{u i}, R_{N(u, i)}\right)\right)
$$

where $\mathscr{R}_{\neg u i}$ is all ratings except for $r_{u i}, N(u, i)$ is the set of neighbors of the pair $(u, i)$ (e.g., see the shaded row and column in Fig. 2), and

$$
E\left(r_{u i}, R_{N(u, i)}\right)=-\left(\log \phi_{u i}\left(r_{u i}\right)+\sum_{(u, j) \in N(u, i)} \log \psi_{i j}\left(r_{u i}, r_{u j}\right)+\sum_{(v, i) \in N(u, i)} \log \varphi_{u v}\left(r_{u i}, r_{v i}\right)\right)
$$

\subsubsection{Rate prediction}

Similar to the case of separate MRFs in Sec. 2.3, rate prediction for a new user/item pair is based on the existing ratings associated with the user and the item as follows:

$$
r_{v j}^{*}=\arg \max _{r_{v j}} P\left(r_{v j} \mid R_{N(v, j)}\right)=\arg \min _{r_{v j}} E\left(r_{v j}, R_{N(v, j)}\right)
$$

where $E\left(r_{v j}, R_{N(u, j)}\right)$ is local energy computed as in Eq. 10 . The expected rating can be computed as:

$$
\bar{r}_{v j}=\sum_{k=1}^{K} P\left(r_{v j} \mid R_{N(u, j)}\right) k
$$




\section{Structure learning}

The MRFs presented in Sec. 2.3 require the model structures to be pre-determined by hand. In this section, we present a method to learn the structure from data. We first introduce several parameterization schemes of the potentials (Sec. 3.1) that facilitate structure learning (Sec. 3.2).

\subsection{Log-linear parameterizations}

It is important to note that ratings are not merely discrete but also ordinal. That is, if the true rating is 3, it is better to get closer to 3 in prediction (e.g., 2 and 4 rather than 1 and 5). This is unlike unordered discrete variables, where all the options are a priori equal. We present here three log-linear parameterization schemes to capture this ordinal property: linear-by-linear, Gaussian, and smoothness. In what follows, we will assume that there are $N$ users and $M$ items in the rating database whose values are drawn from the ordered set $\{1,2, . ., K\}$.

\subsubsection{Linear-by-linear parameterization}

The first scheme is a linear-by-linear parameterization in which the potential functions in Eq. (3) have the following forms:

$$
\begin{aligned}
\phi_{u i}\left(r_{u i}=k\right) & =\exp \left(\alpha_{i k}+\beta_{u k}\right) \\
\psi_{i j}\left(r_{u i}, r_{u j}\right) & =\exp \left(\omega_{i j}\left(r_{u i}-\bar{r}_{i}\right)\left(r_{u j}-\bar{r}_{j}\right)\right) \\
\varphi_{u v}\left(r_{u i}, r_{v i}\right) & =\exp \left(w_{u v}\left(r_{u i}-\bar{r}_{u}\right)\left(r_{v i}-\bar{r}_{v}\right)\right)
\end{aligned}
$$

where $\left\{\alpha_{i k}, \beta_{u k}\right\}$ are rating biases for item $i$ and user $u$, respectively; $\left\{\omega_{i j}, w_{u v}\right\}$ are pairwise interaction parameters for item pair $(i, j)$ and user pair $(u, v)$, respectively; and $\left\{\bar{r}_{i}, \bar{r}_{u}\right\}$ are mean rates for item $i$ and user $u$, respectively. The bias $\alpha_{i k}$ reflects the overall quality of an item, regardless of the user. For example, popular movies tend to receive higher ratings than average. The bias $\beta_{u k}$ indicates the tendency that a user chooses a particular rating. This is because some users may or may not be critical in their rating, and some users only rate items that they like, ignoring those they do not like.

The pairwise potential $\psi_{i j}\left(r_{u i}, r_{u j}\right)$ reflects the ordering of both $r_{i}$ and $r_{j}$. Since its log is linear in either variable, this parameterization is called linear-by-linear model [1, Chap. 8]. This parameterization have $N K+M K+\frac{1}{2} M(M-1)$ parameters. Similar properties hold for $\varphi_{u v}\left(r_{u i}, r_{v i}\right)$. 
Remark. The pairwise potential can be parameterized differently, e.g., $\psi_{i j}\left(r_{i}=k_{1}, r_{j}=k_{2}\right)=\exp \left(\omega_{i j k_{1} k_{2}}\right)$. However, since this multiplies the number of parameters by a factor of $K^{2}$, it is expensive to compute and less reliable to learn. Thus we do not investigate this option in the paper. The main drawback of this approach is the treatment of ordinal ratings as categorical, and thus losing important information.

\subsubsection{Gaussian parameterization}

An approximation to ordinal treatment is the Gaussian parameterization scheme, where ratings are considered as continuous variables. The potential functions in Eq. (3) can be specified as:

$$
\begin{aligned}
\phi_{u i}\left(r_{u i}\right) & =\exp \left(-\frac{\left(r_{u i}-\alpha_{i}-\beta_{u}\right)^{2}}{2}\right) \\
\psi_{i j}\left(r_{u i}, r_{u j}\right) & =\exp \left(\omega_{i j} r_{u i} r_{u j}\right) \\
\varphi_{u v}\left(r_{u i}, r_{v i}\right) & =\exp \left(w_{u v} r_{u i} r_{v i}\right)
\end{aligned}
$$

Thus $\psi_{i j}\left(r_{u i}, r_{u j}\right)$ captures the linear association between items, similar to the linear-by-linear parameterization in Sec. 3.1.1 This parameterization have $N+M+\frac{1}{2} M(M-1)$ parameters. Similar properties hold for $\varphi_{u v}\left(r_{u i}, r_{v i}\right)$.

Remark. The model is log-linear because $\phi_{u i}\left(r_{u i}\right) \propto \exp \left(0.5\left\{-r_{u i}^{2}+\left(\alpha_{i}+\beta_{u}\right) r_{u i}\right\}\right)$ which is log-linear in $\alpha_{i}, \beta_{u}$, and $\psi_{i j}\left(r_{u i}, r_{u j}\right)$ and $\varphi_{u v}\left(r_{u i}, r_{v i}\right)$ are log-linear in $\omega_{i j}$ and $w_{u v}$, respectively.

The local predictive distribution has the following form:

$$
P\left(r_{u j} \mid r_{N(j)}\right) \propto \exp \left(-\frac{\left(r_{j}-\alpha_{j}-\sum_{i \in N(j)} \omega_{i j} r_{u i}\right)^{2}}{2}\right)
$$

When $r_{u j}$ is allowed to take value in entire $\mathbb{R}$, it is essentially a normal distribution of mean $\alpha_{j}+$ $\sum_{i \in N(j)} \omega_{i j} r_{u i}$.

Rate normalization. The Gaussian model assumes ratings of variance 1. It necessitates normalization before training can start. Our normalization is a two-step procedure:

1. The first step normalizes data per user. Adapting from [11], we transform the rating as follows

$$
\hat{r}_{u i} \leftarrow \frac{r_{u i}-\bar{r}_{u}}{\bar{s}_{u}},
$$


where $\bar{r}_{u}$ and $\bar{s}_{u}$ are the mean rating and smoothed deviation by user $u$, respectively. The smoothed deviation is estimated from the deviation $s_{u}$ as follows:

$$
\bar{s}_{u}=\sqrt{\frac{5 s^{2}+n_{u} s_{u}^{2}}{5+n_{u}}},
$$

where $s$ is the global deviation for the whole train data, $n_{u}$ is the number of items rated by user $u$. Thus $\bar{s}_{u}$ is between $s$ and $s_{u}-\bar{s}_{u}$ is closer to $s$ if $n_{u}$ is small, and to $s$ otherwise.

2. The second step normalizes data per item.

$$
\hat{\hat{r}}_{u i} \leftarrow \frac{\hat{r}_{u i}-\overline{\hat{r}}_{i}}{\overline{\hat{s}}_{i}},
$$

where $\overline{\hat{r}}_{i}$ is the mean rate for item $i$ after the first step and $\overline{\hat{s}}_{i}$ is the smoothed deviation computed as:

$$
\overline{\hat{s}}_{i}=\sqrt{\frac{5+\sum_{u \in U(i)}\left(\hat{r}_{u i}-\overline{\hat{r}}_{i}\right)}{5+m_{i}}} .
$$

Thus $\overline{\hat{s}}_{i}$ is closer to 1 if $m_{i}$ is small.

At prediction time, the reverse process is performed to recover the original scale:

$$
r_{u i} \leftarrow \bar{r}_{u}+\bar{s}_{u}\left(\overline{\hat{r}}_{i}+\overline{\hat{s}}_{i} \hat{\hat{r}}_{u i}\right)
$$

\subsubsection{Smoothness parameterization}

While Gaussian parameterization respects the ordinal property of ratings, the Gaussian assumption could be too strong. In this approach we employ an ordinal parameterization following [25, 26]:

$$
\begin{aligned}
\phi_{u i}\left(r_{u i}=k_{1}\right) & =\exp \left(-\sum_{k_{2}=1}^{K}\left(\alpha_{i k_{2}}+\beta_{u k_{2}}\right)\left|k_{1}-k_{2}\right|\right) \\
\psi_{i j}\left(r_{u i}, r_{u j}\right) & =\exp \left(-\omega_{i j}\left|r_{u i}-r_{u j}\right|\right) \\
\varphi_{u v}\left(r_{u i}, r_{v i}\right) & =\exp \left(-w_{u v}\left|r_{u i}-r_{v i}\right|\right)
\end{aligned}
$$

This parameterization have $N K+M K+\frac{1}{2} M(M-1)$ parameters. The singleton potential $\phi_{u i}\left(r_{u i}\right)$ captures the relative distances of the current rating from anchor points $\{1,2, . ., K\}$. The pairwise potential $\psi_{i j}\left(r_{u i}, r_{u j}\right)$ enables smoothness between neighbor ratings by the same user, parameterized by $\omega_{i j}$. A similar property holds for $\varphi_{u v}\left(r_{u i}, r_{v i}\right)$ for the same item. 


\subsection{Structure learning for user-specific models}

Given our log-linear parameterizations, an edge contributes to model energy only if its parameter is non-zero. Thus, structure learning reduces to estimating non-zero pairwise parameters $\left\{\omega_{i j}\right\}$ and $\left\{w_{u v}\right\}$. For clarity, let us start with user-specific models (Sec. 2.3. We propose to minimize the following $\ell_{1}$-penalized loss:

$$
\theta^{*}=\arg \min _{\theta}\left(\mathscr{L}(\theta)+\lambda_{1} \sum_{i} \sum_{j>i}\left|\omega_{i j}\right|\right)
$$

where $\theta=(\alpha, \beta, \omega)$ denotes the set of model parameters, $\mathscr{L}(\theta)$ is the loss function, and $\lambda_{1}>0$ is the regularization parameter. This setting has the following properties:

- The $\ell_{1}$-penalty drives weakly weights of correlated item pairs towards zero, thus achieving a sparse item graph solution as a by-product, and

- The hyper-parameter $\lambda_{1}$ controls the sparsity of the solution, that is, the higher $\lambda_{1}$ leads to more sparse solutions.

A typical loss function is the negative $\log$-likelihood, i.e., $\mathscr{L}(\theta)=-\log P(r ; \theta)$, where $P(r ; \theta)$ is defined in Eq. (3). When the gradient of the loss function is available, we can use the gradient ascent method to optimize it. For example, the gradient descent update for the pairwise parameters is

$$
\omega_{i j} \leftarrow \omega_{i j}-\eta\left(\partial_{\omega_{i j}} \mathscr{L}(\theta)+\lambda_{1} \operatorname{sign}\left(\omega_{i j}\right)\right)
$$

where $\eta>0$ is the learning rate. However, since $P(r ; \theta)$ and the gradient $\partial_{\omega_{i j}} \mathscr{L}(\theta)$ are intractable to compute exactly, we resort to surrogate methods - one approximates the loss (pseudo-likelihood), the other approximates the gradient (contrastive divergence). While there are many algorithms to deal with non-smooth gradient due to the step function $\operatorname{sign}()$, we employ here a simple approximate solution:

$$
\left|\omega_{i j}\right| \approx \sqrt{\varepsilon^{2}+\omega_{i j}^{2}}
$$

for $0<\varepsilon \ll 1$, which has the smooth gradient $\left[\varepsilon^{2}+\omega_{i j}^{2}\right]^{-1 / 2} \omega_{i j}$.

\subsubsection{Pseudo-likelihood (PL)}

The pseudo-likelihood loss is defined in Eq. (8). The loss and its gradient can be computed exactly. For example, for Gaussian parameterization, the gradient for pairwise parameters is

$$
\partial_{\omega_{i j}} \mathscr{L}_{P L}(\theta)=\mu_{i} r_{u j}+\mu_{j} r_{u i}-2 r_{u i} r_{u j}
$$


where

$$
\mu_{i}=\alpha_{i}+\sum_{j_{1} \in N(i)} \omega_{i j_{1}} r_{u j_{1}}
$$

\subsubsection{Contrastive divergence $(C D)$}

Alternatively, we use the original loss function, but approximate its gradient. For example, for Gaussian parameterization, the derivative with respect to pairwise parameters reads:

$$
\partial_{\omega_{i j}} \mathscr{L}(\theta)=\mathbb{E}\left[r_{u i} r_{u j}\right]-r_{u i} r_{u j}
$$

The expectation $\mathbb{E}\left[r_{u i} r_{u j}\right]$ can be approximated by samples drawn from the distribution $P(r)$ as follows:

$$
\mathbb{E}\left[r_{u i} r_{u j}\right] \approx \frac{1}{n} \sum_{s=1}^{n} r_{u i}^{(s)} r_{u j}^{(s)}
$$

Since full sampling until convergence is expensive, we employ a short-cut called $c$-step contrastive divergence [10] (CD). More specifically, we start a Markov chain from the data $r$ itself, and apply Gibbs sampling for $c$ scans over all items. The Gibbs sampling iteratively draws a rating at a time using $r_{u i}^{(s)} \sim P\left(r_{u i} \mid r_{\neg u i}^{(s)}\right)$, updating the samples along the way. Typically $c$ is a small number. The sample at the end of the $c$ scans will be retained to approximate the gradients.

\subsubsection{Reducing computational complexity}

The prediction complexity per item is $\mathscr{O}\left(m_{\max } K\right)$ for the linear-by-linear and Gaussian models and $\mathscr{O}\left(m_{\max } K^{2}\right)$ the smoothness model, where $m_{\max }$ is the maximum number of items per users. Typically, this is fast because $m_{\max } \ll M$.

In learning, we use mini-batches of $b$ users to compute gradients to speed up. At each parameter update, learning takes $\mathscr{O}\left(\left(m_{\max }+m_{\max }^{2}\right) b K\right)$ time to compute gradient and $\mathscr{O}\left((N+M) K+\frac{1}{2} M^{2}\right)$ time to update parameters. Memory consumes $\mathscr{O}\left((M+N) K+\frac{1}{2} M^{2}\right)$ space to store parameters. We propose to reduce these demanding memory and time by specifying the max number of neighbors, e.g., $m \ll M$, giving a time complexity of $\mathscr{O}((M+N) K+M m)$. One way to specify the neighbor is pre-filtering by correlation measures, that is, we keep only highly correlated item pairs. However, this method creates a significant run-time overhead of $\mathscr{O}(\log m)$ by checking if a pair is pre-selected. In this paper, we use a simple method for fast memory access: each item is connected to $m$ most popular items. 


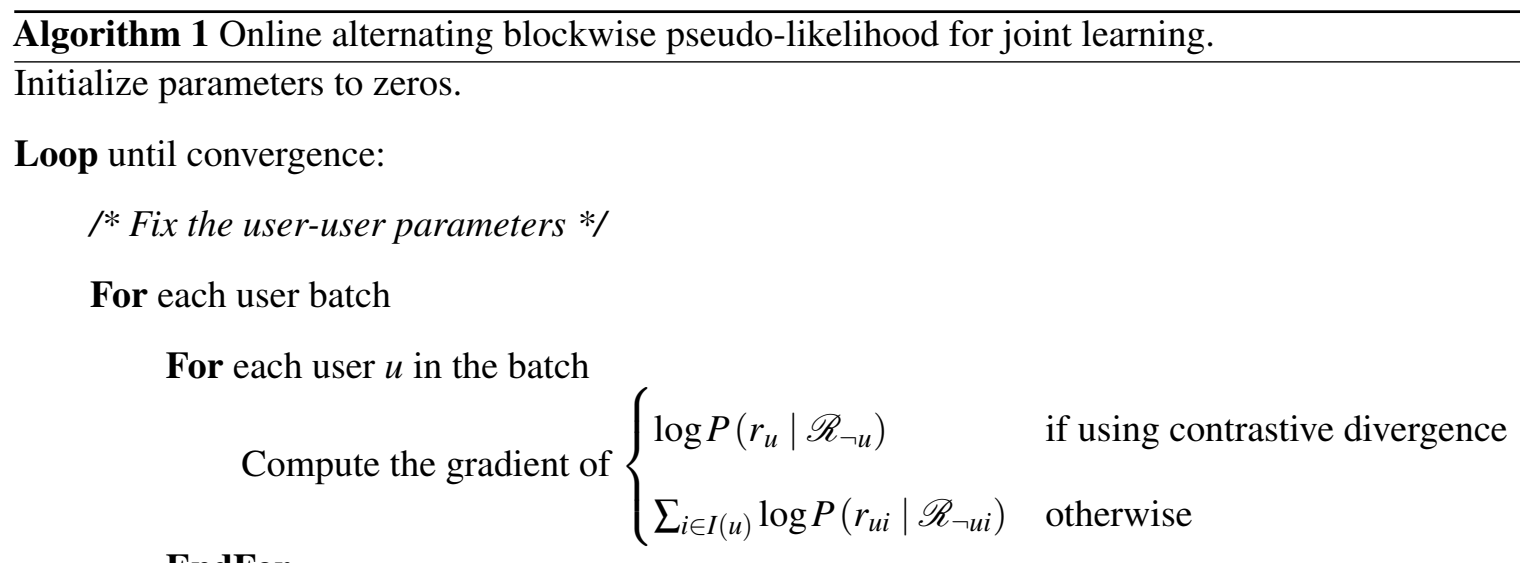

\section{EndFor}

Update relevant biases $(\alpha, \beta)$ and item-item pairwise parameters $\omega$

\section{EndFor}

/* Fix the item-item parameters */

For each item batch

For each item $i$ in the batch

$$
\text { Compute the gradient of } \begin{cases}\log P\left(r_{i} \mid \mathscr{R}_{\neg i}\right) & \text { if using contrastive divergence } \\ \sum_{u \in U(i)} \log P\left(r_{u i} \mid \mathscr{R}_{\neg u i}\right) & \text { otherwise }\end{cases}
$$

\section{EndFor}

Update biases $(\alpha, \beta)$ and user-user pairwise parameters $w$

\section{EndFor}

\section{EndLoop}

\subsection{Structure learning of the entire rating database}

Extension to the entire rating database of Sec. 2.4 is straightforward. Eq. (22) is now extended to:

$$
\theta^{*}=\arg \min _{\theta}\left(\mathscr{L}(\theta)+\lambda_{1} \sum_{i} \sum_{j>i}\left|\omega_{i j}\right|+\lambda_{2} \sum_{u} \sum_{v>u}\left|w_{u v}\right|\right)
$$

where $\theta$ now consists of all user-specific and item-specific parameters, $\omega_{i j}$ is item-item parameter, $w_{u v}$ is the user-user parameter, and $\lambda_{1}, \lambda_{2}>0$.

We present an efficient algorithm that relies on blockwise pseudo-likelihood. For example, an user's rating set is a block from which the conditional distribution $P\left(r_{u} \mid \mathscr{R}_{\neg u}\right)$ is estimated, where $\mathscr{R}_{\neg u}$ is the set of all ratings except for those by user $u$. Likewise, we also have an item block with the conditional 
distribution $P\left(r_{i} \mid \mathscr{R}_{\neg i}\right)$, where $\mathscr{R}_{\neg i}$ is the set of all ratings except for those on item $i$. Thus it is an extension of the standard pointwise pseudo-likelihood in [3].

This suggests an alternating procedure between updating user-based models and item-based models. For each model, the learning techniques (pointwise pseudo-likelihood and contrastive divergence) presented in Sec. 3.2 are applicable. When computing $P\left(r_{u} \mid \mathscr{R}_{\neg u}\right)$, we need to take the user neighborhoods of $r_{u i}$ for each item $i$ rated by user $u$. Likewise, when computing $P\left(r_{i} \mid \mathscr{R}_{\neg i}\right)$, we need to account for the item neighborhoods of $r_{u i}$ for each user $u$ who rated item $i$.

The overall algorithm is presented in Alg. 1. It is an online-style algorithm for speeding up. In particular, we update parameters after every small batch of either users or items.

\subsubsection{Reducing computational complexity}

Similar to those described in Sec. 3.2.3 we use the method of limiting neighborhood size to $m \ll M$ items and $n \ll N$ users. The prediction complexity per item is $\mathscr{O}((n+m) K)$ for linear-bylinear (Sec. 3.1.1) and Gaussian (Sec. 3.1.2 parameterizations and $\mathscr{O}\left((n+m) K^{2}\right)$ for the smoothness parameterization (Sec. 3.1.3). At each parameter update after a batch of size $b$, learning takes $\mathscr{O}\left(\left(n+m+n^{2}+m^{2}\right) b K\right)$ time to compute gradient for linear-by-linear and Gaussian parameterizations and $\mathscr{O}\left(\left(n+m+n^{2}+m^{2}\right) b K^{2}\right)$ for the smoothness parameterization. Parameter update takes $\mathscr{O}((N+M) K+n N+m M)$ time for all parameterizations.

\section{Experimental results}

In this section, we present a comprehensive evaluation of our proposed method on two applications: movie recommendation and online date matching.

\subsection{Experimental setup}

For rating prediction, we report three measures: the root-mean square error (RMSE), the mean absolute error (MAE) and the log-likelihood (LL). 


$$
\begin{aligned}
R M S E & =\sqrt{\frac{1}{Y} \sum_{u=1}^{N} \sum_{j \in J(u)}\left(r_{u j}-\bar{r}_{u j}\right)^{2}} \\
M A E & =\frac{1}{Y} \sum_{u=1}^{N} \sum_{j \in J(u)}\left|r_{u j}-r_{u j}^{*}\right| \\
L L & =\frac{1}{Y} \sum_{u=1}^{N} \sum_{j \in J(u)} \log P\left(r_{u j}\right)
\end{aligned}
$$

where $J(u)$ is the set of new items for user $u, \bar{r}_{u j}$ is the expected rating from Eqs. $7\left[12, r_{u j}^{*}\right.$ is predicted rating from Eqs. (6 11), and $Y$ is total number of new predictions (i.e., $Y=\sum_{u=1}^{N} \sum_{j \in J(u)} 1$ ). The RMSE and MAE measure the distance from the predicted rating and the true rating (i.e., the smaller the better). The log-likelihood, on the hand, measures how the model fits the unseen data (i.e., the larger the better).

\subsubsection{MRF implementation}

Learning for MRFs is iterative in that for each iteration, first user-specific models are updated followed by item-specific models. Parameters are updated after every batch of $b=100$ users or items. Learning rate $\eta$ is set at 0.1 for biases and 0.01 for pairwise parameters. To speed up, at the early learning stages, only biases are learnt. Once the learning starts getting saturated, pairwise parameters are then introduced. Note that this schedule does not alter the solution quality since the objective function is concave. But it may improve the convergence speed because pairwise gradients are much more expensive to estimate. Rate prediction is by Eq. (6) when an integer output is expected (e.g., for estimating MAE), and by Eq. (7) for a real-valued output (e.g., for estimating RMSE).

\subsubsection{Baselines}

For comparison we implemented 3 simple baselines, one using user mean-rating $\bar{r}_{u}$ as prediction for user $u$, another using item mean-rating $\bar{r}_{i}$ for item $i$, and a weighted mean accounting for variances:

$$
\bar{r}_{u i}=\frac{\bar{r}_{u} / s_{u}+\bar{r}_{i} / s_{i}}{1 / s_{u}+1 / s_{i}}
$$

where $s_{u}$ and $s_{i}$ are rating deviations for user $u$ and item $i$, respectively.

We also implemented one of the best performing techniques in the Netflix challenge: regularized singular value decomposition (RSVD), also known as probabilistic matrix factorization [20]. They are 
both latent space models. The RSVD assumes a generative Gaussian distribution of ratings:

$$
\begin{aligned}
r_{u i} & \sim \mathscr{N}\left(\mu_{u i}, \sigma_{u i}^{2}\right) \\
\mu_{u i} & =a_{i}+b_{u}+\sum_{f=1}^{F} A_{i f} B_{u f} \\
\sigma_{u i}^{2} & =e^{\gamma_{u}+v_{i}} \\
A_{i f} & \sim \mathscr{N}\left(0, \lambda^{-1}\right) \\
B_{u f} & \sim \mathscr{N}\left(0, \lambda^{-1}\right)
\end{aligned}
$$

Here $\left\{a_{i}, b_{u}, A_{i f}, B_{u f}, \gamma_{u}, v_{i}\right\}$ are free parameters and $F$ is the latent dimensions. Unlike existing RSVD implementation where $\sigma_{u i}^{2}=1$, we also estimate the variance $\sigma_{u i}^{2}$ for the purpose of estimating a better data likelihood. The prior variance $\lambda$ is tuned for the best performance.

\subsubsection{Validation}

For each dataset, we remove those infrequent users who have less than 30 ratings. Then 5 items per user are held out for validation, 10 for testing and the rest for training. We make sure that the prediction is time-sensible, i.e., the training time-stamps precedes validation which precedes testing. For MRFs, learning is monitored using the pseudo-likelihood on the validation data. Learning is stopped if there is no improvement of pseudo-likelihood on the validation set.

\subsection{Movie recommendation}

For movie recommendation, we use the MovieLens $1 \mathrm{M}^{1}$ dataset with 1 million ratings in a 5 -star scale given by 6 thousand users on approximately 4 thousand movies. After removing infrequent users, we retain 5.3 thousand users, 3.3 thousands items and 901.1 thousand ratings. The mean rating is 3.6 (std: 1.1) and the rating matrix is sparse, with only 5.2\% cells filled. On average, a movie is rated 276 times (median: 145), and an user rates 170 movies (median: 99).

\subsubsection{Learning curves}

Fig. 3 shows typical learning curves. There is a gap between the pseudo-likelihoods on training and validation data. The gap is widen as soon as pairwise parameters are introduced, suggesting that there is a chance of overfitting. Thus the $\ell_{1}$-regularization and validation for early stopping are essential.

\footnotetext{
${ }^{1}$ http://www.grouplens.org/node/12
} 

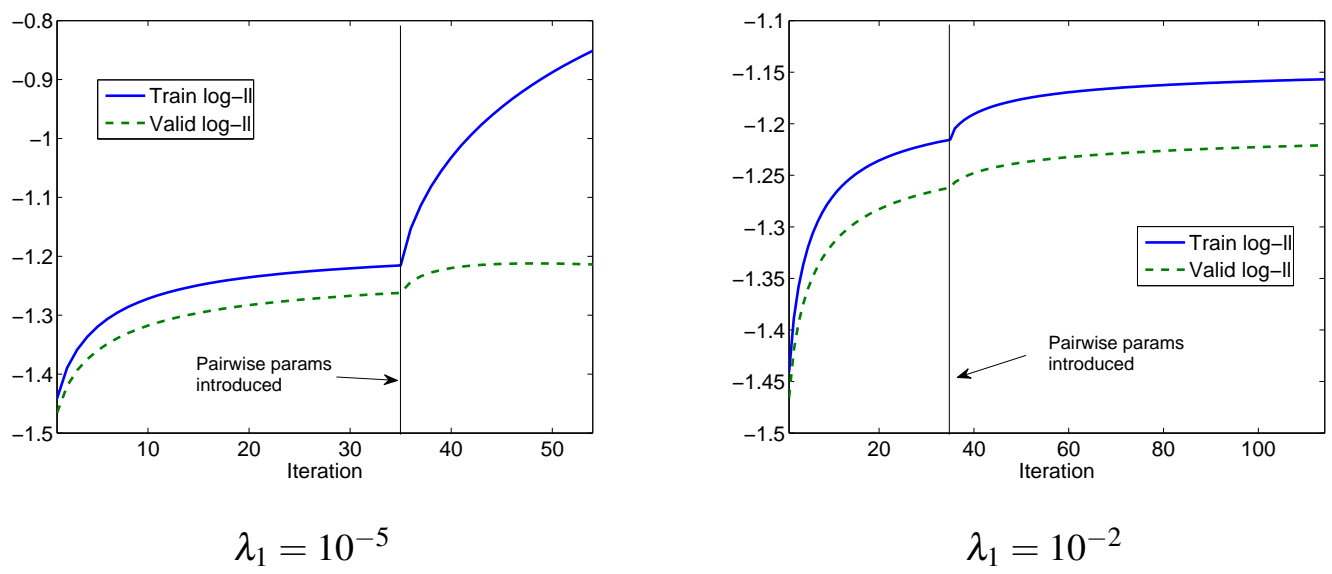

Figure 3: Learning curves for pseudo-likelihood. Data: MovieLens 1M; model: user-specific with smoothness parameterization (Sec. 3.1.3) learnt from pseudo-likelihood (Sec. 3.2.1). There are two stages: first only biases are learnt, then pairwise parameters are introduced. $\lambda_{1}$ is the sparsity inducing factor as in Eq. (22). The gap between the training log-likelihood and the validation indicates potential overfitting.

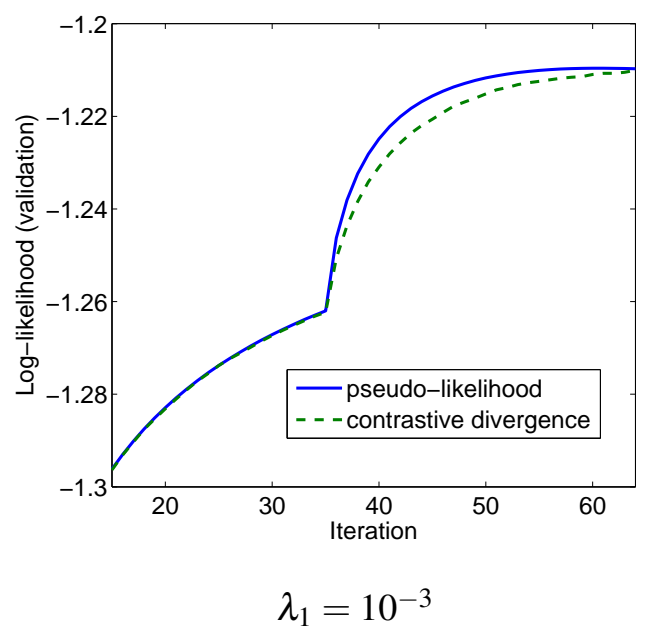

Figure 4: Learning curves for pseudo-likelihood (PL, Sec. 3.2.1) versus contrastive divergence (CD, Sec. 3.2.2). Data: MovieLens 1M; model: user-specific with smoothness parameterization (Sec. 3.1.3. 


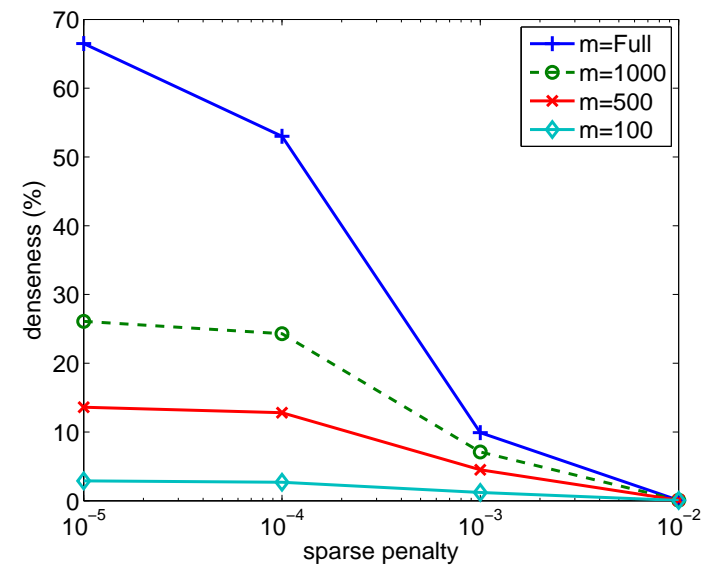

Figure 5: Sparsity controlled by the $\ell_{1}$-penalty $\lambda_{1}$. Data: MovieLens $1 \mathrm{M}$; model: user-specific with smoothness parameterization (Sec. 3.1.3) learnt from pseudo-likelihood (Sec. 3.2.1).

Increasing the penalty factor from $\lambda_{1}=10^{-5}$ (Fig. 3-left) to $\lambda_{1}=10^{-2}$ (Fig. 3-right) helps tremendously in combating against overfitting. Fig. 4 depicts comparison between pseudo-likelihood (Sec. 3.2.1) and contrastive divergence (Sec. 3.2.2). Overall these two learning methods behave similarly, with pseudo-likelihood produces a slightly faster convergence. For the rest of the section, we will report the results for pseudo-likelihood training only unless specified otherwise.

\subsubsection{Sensitivity analysis}

The sparsity of the graphs are measured as the ratio of number of non-zeros edges and number of fully connected edges. Fig. 5 represents graph sparsity against the $\ell_{1}$-penalty $\lambda_{1}$ and the max neighborhood size $m$ (see Sec. 3.2.3). Larger penalty and smaller neighborhood size lead to more sparsity (equivalently, less denseness). However, the two hyperparameters $\lambda_{1}$ and $m$ do affect the performance. For fully connected item graphs, top performance is reached at $\lambda_{1}=10^{-3}$ on the MovieLens $1 \mathrm{M}$ data, achieving a sparsity of $9.9 \%$. For smaller neighborhoods, $\lambda_{1}=10^{-4}$ is the best setting. Overall, the performance depends more on the sparsity penalty, and less on the neighborhood size. It is desirable because it allows significant reduction of memory footprint, which is proportional to $m$, with little loss of accuracy.

To verify whether the MRF can work with limited data, we randomly pick $q$ ratings per user in the training data. Fig. 7 depicts the behavior of the MRF with the smoothness parameterization when $q=\{10,20$, full $\}$. The behavior is consistent with the expectation that more data would lead to better 

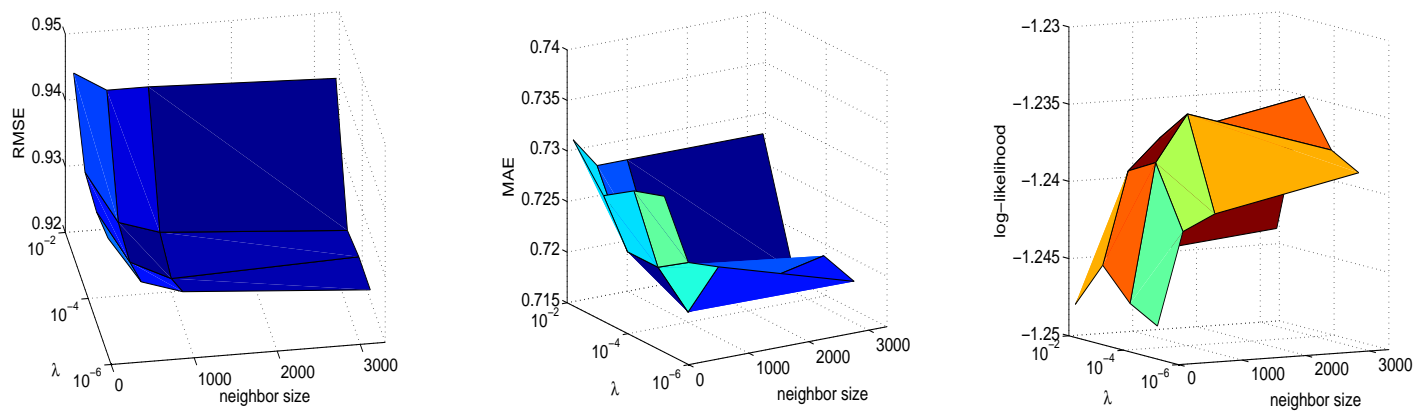

Figure 6: Performance sensitivity against hyperparameters: $m$ - max size of neighborhood, and $\lambda_{1}$ - the $\ell_{1}$-penalty in Eq. 22). Data: MovieLens 1M; model: user-specific with smoothness parameterization (Sec. 3.1.3 learnt from pseudo-likelihood (Sec. 3.2.1 (Best viewed in color).

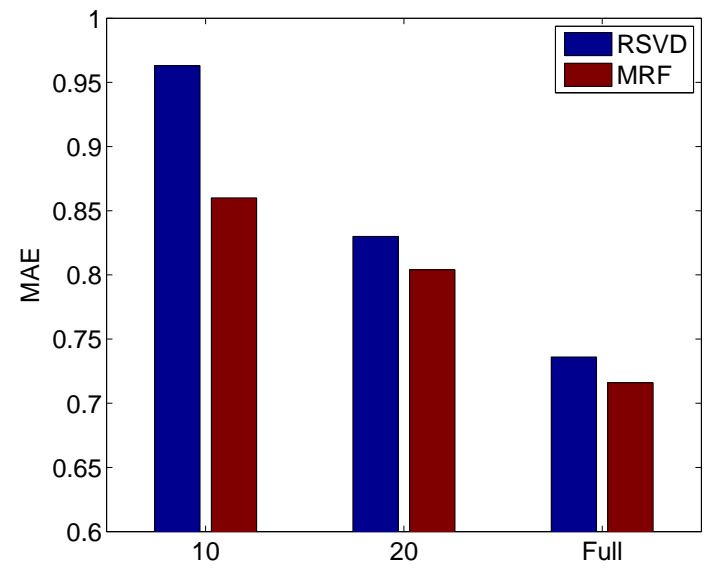

Figure 7: Mean Absolute Error (MAE) as function of training size per user, $\lambda_{1}=10^{-3}$ (the smaller MAE the better). Data: MovieLens 1M; model: user-specific with smoothness parameterization (Sec. 3.1.3 learnt from pseudo-likelihood (Sec. 3.2.1p (Best viewed in color). 


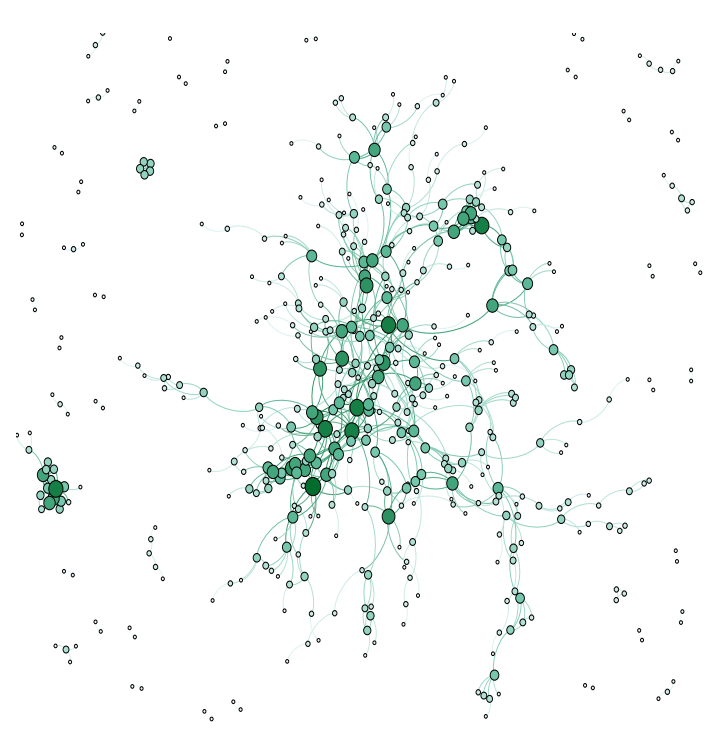

(a) Positive correlation

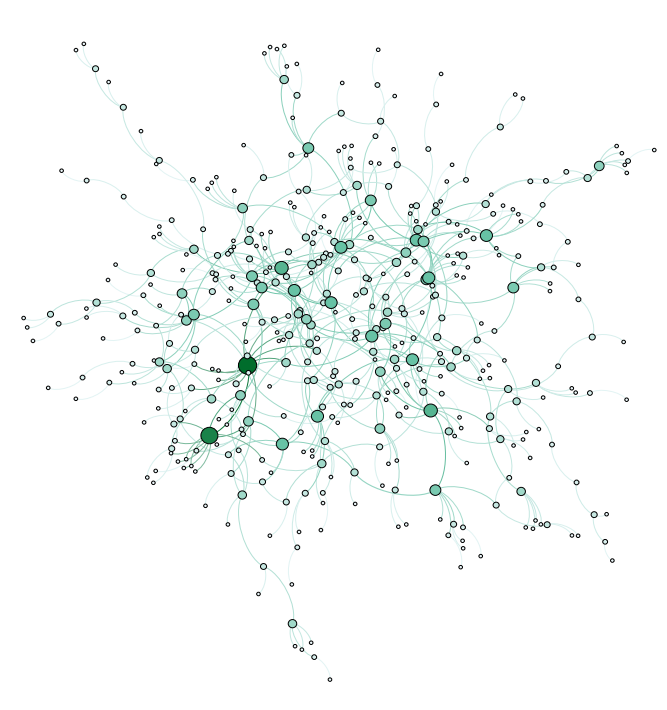

(a) Negative correlation

Figure 8: Movie graphs $\left(\lambda_{1}=\lambda_{2}=10^{-3}\right.$, smoothness parameterization, contrastive divergence training). Node size represents connectivity degree. To prevent clutters, only strong correlations are shown.

performance. Importantly, compared against the RSVD, it shows that the MRF is more robust against small data.

\subsubsection{User and movie graphs}

Our $\ell_{1}$-regularization framework in Eq. 25] naturally discovers item correlation and user correlation graphs. As previous demonstrated in Fig. 5, by varying the penalty hyperparameters $\lambda_{1}$ (to control item-item graph sparsity) and $\lambda_{2}$ (to control user-user graph sparsity), we obtain different connectivity patterns with varying degree of sparsity. Figs. $8(a, b)$ plot movie graphs estimated from the MovieLens 1M data. Positively correlated movies are those liked (or disliked) in similar ways, while a negative correlation means the two movies received diverse opinions. The user graphs shown in Figs. 9 appear to have a handful of users who have a high degree of connectivity, either positively (agreement) or negatively (disagreement). This fact could be exploited to locate influential users in social networks.

\subsubsection{Model performance}

Tab. 1 reports the results on the test data for various model settings. The MRF's performance consistently improves when user models and item models are joined. The Gaussian parameterization (with careful normalization described in Sec. 3.1.2) achieves the best fit in term of data log-likelihood 


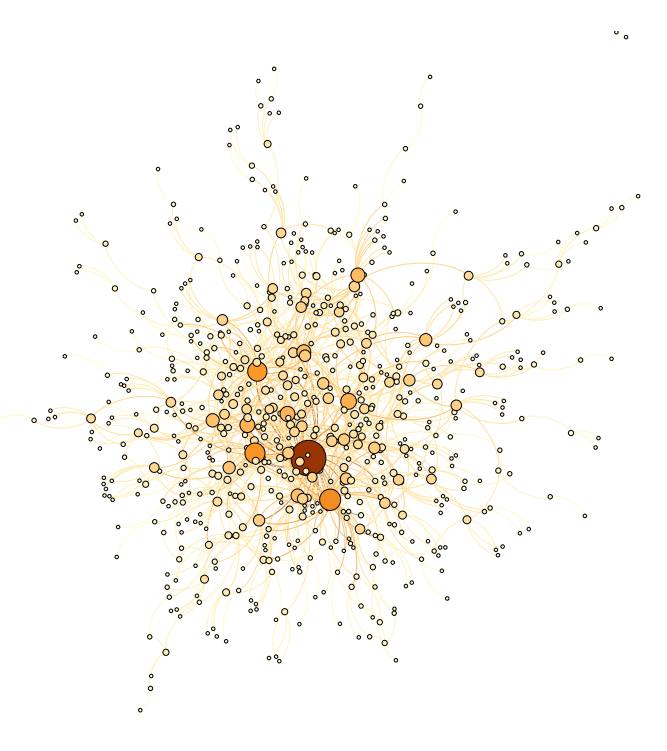

(a) Positive correlation

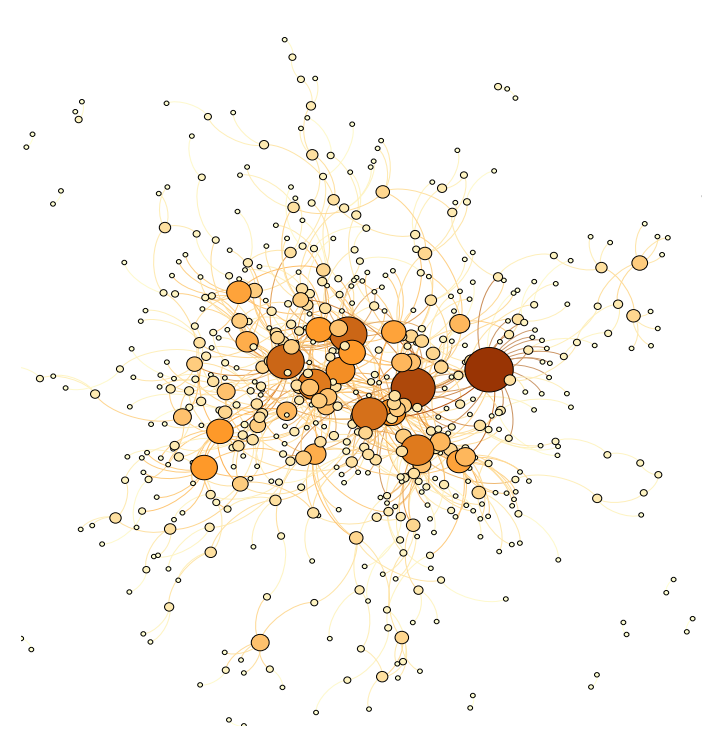

(a) Negative correlation

Figure 9: User graphs on movie data $\left(\lambda_{1}=\lambda_{2}=10^{-3}\right.$, smoothness parameterization, contrastive divergence training). Node size represents connectivity degree. To prevent clutters, only strong correlations are shown.

(LL). All MRF parameterizations excel on MAE and LL measures. The best performance in RMSE and MAE are smoothness parameterization.

\subsection{Date matching}

For the date matching task, the dataset is Dating Agency $\left.\right|^{2}$ with 17 million ratings in a 10-point scale by 135 thousand users who rated nearly 169 thousand profiles. To make the experiments comparable with those in movie recommendation, we rescale the ratings of the Dating Agency to the 5-point scale. After processing, we retain 101.3 thousand users, 34.9 thousand items and 12.5 million ratings. The mean rating is 1.9 (std: 1.5 ) and the ratings are quite uniformly distributed. The rating matrix is $0.4 \%$ dense, meaning that only $0.4 \%$ profiles are rated on average. An average user rates 123 profiles but median is only 73, indicating a skew toward small rating history. An item is rated 357 times on average (median: 192). As the number of users and profiles are large, it is necessary to limit the neighborhood size to $m \ll M$ for manageable memory footprint. Other than that we use the same settings as in the movie recommendation experiments.

\footnotetext{
${ }^{2} \mathrm{http}: / /$ www.occamslab.com/petricek/data/
} 


\begin{tabular}{|lccc|}
\hline Method & $R M S E$ & $M A E$ & $L L$ \\
\hline User-mean & 1.094 & 0.865 & - \\
Item-mean & 1.006 & 0.806 & - \\
Weighted-mean & 0.992 & 0.792 & - \\
RSVD $(F=50)$ & 0.932 & 0.736 & -1.353 \\
RSVD $(F=100)$ & 0.921 & 0.729 & -1.311 \\
\hline MRF.user.Gauss.PL & 0.930 & $\mathbf{0 . 7 2 8}$ & $\mathbf{- 1 . 1 8 9}$ \\
MRF.item.Gauss.PL & 0.929 & $\mathbf{0 . 7 2 6}$ & $\mathbf{- 1 . 1 8 8}$ \\
MRF.joint.Gauss.PL & 0.929 & $\mathbf{0 . 7 2 6}$ & $\mathbf{- 1 . 1 8 8}$ \\
\hline MRF.user.linear-linear.PL & 0.940 & $\mathbf{0 . 7 2 4}$ & $\mathbf{- 1 . 2 4 9}$ \\
MRF.item.linear-linear.PL & 0.942 & $\mathbf{0 . 7 2 0}$ & $\mathbf{- 1 . 2 5 3}$ \\
MRF.joint.linear-linear.PL & 0.931 & $\mathbf{0 . 7 0 8}$ & $\mathbf{- 1 . 2 4 3}$ \\
\hline MRF.user.smooth.PL & 0.922 & $\mathbf{0 . 7 1 6}$ & $\mathbf{- 1 . 2 3 5}$ \\
MRF.item.smooth.PL & $\mathbf{0 . 9 2 0}$ & $\mathbf{0 . 7 1 2}$ & $\mathbf{- 1 . 2 2 9}$ \\
MRF.joint.smooth.PL & $\mathbf{0 . 9 1 2}$ & $\mathbf{0 . 7 0 3}$ & $\mathbf{- 1 . 2 1 8}$ \\
\hline MRF.user.smooth.CD & 0.922 & $\mathbf{0 . 7 1 6}$ & $\mathbf{- 1 . 2 3 4}$ \\
MRF.user.smooth.CD & $\mathbf{0 . 9 1 4}$ & $\mathbf{0 . 7 0 4}$ & $\mathbf{- 1 . 2 2 1}$ \\
MRF.joint.smooth.CD & $\mathbf{0 . 9 1 0}$ & $\mathbf{0 . 7 0 2}$ & $\mathbf{- 1 . 2 1 6}$ \\
\hline
\end{tabular}

Table 1: Rating prediction for MovieLens $1 \mathrm{M}, m=M$ and $\lambda_{1}=\lambda_{2}=10^{-3} . F$ is the number of hidden dimensions in the RSVD. Legend: RMSE - root mean squared error; MAE - mean absolute error; LL data log-likelihood; user - user-specific model; item - item-specific model; joint - joint model; PL pseudo-likelihood; and CD - contrastive divergence. Bolds indicate that the MRF-based results are better than the baselines. 


\begin{tabular}{|l|lllc|}
\hline Method & $m$ & $R M S E$ & $M A E$ & $L L$ \\
\hline User-mean & - & 1.377 & 1.159 & - \\
Item-mean & - & 0.932 & 0.654 & - \\
Weighted-mean & - & 0.955 & 0.743 & - \\
RSVD $(F=50)$ & - & 0.886 & 0.616 & -1.084 \\
RSVD $(F=100)$ & - & 0.895 & 0.624 & -1.096 \\
\hline MRF.user.Gauss.PL & 1,000 & $\mathbf{0 . 8 6 2}$ & $\mathbf{0 . 6 1 5}$ & $\mathbf{- 1 . 1 5 0}$ \\
MRF.user.linear-linear.PL & 1,000 & $\mathbf{0 . 8 4 7}$ & $\mathbf{0 . 5 1 7}$ & $\mathbf{- 0 . 8 5 8}$ \\
MRF.user.smooth.PL & 1,000 & $\mathbf{0 . 8 0 8}$ & $\mathbf{0 . 4 8 0}$ & $\mathbf{- 0 . 8 2 1}$ \\
MRF.user.smooth.PL & 3,000 & $\mathbf{0 . 7 9 3}$ & $\mathbf{0 . 4 6 7}$ & $\mathbf{- 0 . 8 0 7}$ \\
MRF.user.smooth.PL & 5,000 & $\mathbf{0 . 7 8 9}$ & $\mathbf{0 . 4 6 3}$ & $\mathbf{- 0 . 8 0 3}$ \\
\hline
\end{tabular}

Table 2: Rating prediction for Dating Agency data. Bolds indicate that the MRF-based results are better than the baselines. Here: $\lambda_{1}=\lambda_{2}=10^{-5}$.

Tab. 2 reports the results. The simple item mean performs surprisingly well (MAE: 0.654) compared to the more sophisticated method RSVD (MAE: 0.616 with $K=50$ ). As the RMSE essentially captures the variance of each method, the user variance is much higher than item variance. It suggests that users are willing to rate a diverse set of profiles. In addition, profiles receive a high degree of agreement (e.g., with smaller RMSE and MAE, on average).

As with the previous experiments on movie data, the smoothness parameterization leads to the best performance. In particular, with $m=1,000$, the user-specific model achieves a MAE of 0.480 , which is $22.1 \%$ better than the best baseline (RSVD with 50 hidden features). The improvement increases to $24.8 \%$ when $m$ is enlarged to 5,000 . Note that this is still a small neighborhood, .e., $m=1,000$ accounts for only $2.9 \%$ of full item neighborhood.

Unlike the case of MovieLens 1M, the Gaussian parameterization does not fit the data well. Interestingly, the data likelihood is similar to that in the case of MovieLens 1M, regardless of the differences between the two datasets. This could be due to unrealistic distribution assumption about the unit variance and the full real-valued domain for the normalized rating (the domain of the ratings in fact contains only 5 discrete points). 


\section{Conclusion and future work}

This paper focuses on Markov random fields (MRF) as a principled method for modeling a recommender system. We aimed to solve the open problem of structure learning in the MRFs, which happen to be among the largest networks ever studied, with millions of nodes and hundreds of millions of edges. Our solution has two components. One is the log-linear parameterization schemes and the other is a sparsity-inducing framework through $\ell_{1}$-norm regularization. Unlike existing work where model structure must be specified by hand, our framework jointly discovers item-item and user-user networks from data in a principled manner. The density of these networks can be easily controlled by a hyper-parameter. We evaluated the proposal through extensive experiments on two large-scale datasets - the MovieLens 1M with 1 million ratings, the Dating Agency with 17 million ratings.

\subsection{Findings}

The experiments lead to the following findings:

- Compared to state-of-the-art collaborative filtering algorithms, our sparse MRFs have higher performance, and is more robust against small training data.

- There exist optimal sparsity factors $\lambda_{1}$ and $\lambda_{2}$ (see Eq. (25), with respect to prediction accuracy.

- The complexity of the learning algorithm can be significantly reduced by several orders of magnitude through selecting a small neighborhood size with little loss of accuracy.

- Generally, the smoothness parameterization (Sec. 3.1.3) does best in RMSE and MAE.

- For the MovieLens 1M data, the user graphs have the "hubness" characteristic, where there exist several users with high degree of connectivity (e.g., see [24]). They are likely to be the influencers in this social network.

- Finally, a compact and powerful MRF can be estimated efficiently for recommender systems which may involve hundreds of millions of parameters.

\subsection{Limitations and future work}

We observe several limitations which open rooms for future work: 
- The MRFs, while powerful and accurate, are expensive to train, compared to the latent aspects approach RSVD and RBM. We have introduced a way to reduce the complexity significantly by using only popular neighbors without hurting the performance. Further, MRFs can be combined with RSVD in our recent work in [15], and combined with RBM in [26] but the sparse MRFs have not been investigated.

- This paper, like the majority of collaborative filtering literature, assumes that recommendations are first based on rating prediction. While it is reasonable to assume that we should recommend items with potentially high personal rating, it ignores other dimensions such as novelty and diversity. One solution is to use entropy as a measure of novelty:

$$
H(u, i)=-\sum_{r_{u i}} P\left(r_{u i} \mid r\right) \log P\left(r_{u i} \mid r\right)
$$

- Since the attention of the user is limited, it is better to suggest just a few items at a time. As such, item ranking may be more appropriate than rate prediction. Motivated by the expected rating in Eq. (7), we propose to use expected energy decrease as ranking criterion

$$
s_{j}=\sum_{r_{u j}} P\left(r_{u j} \mid r_{N(j)}\right)\left[-E\left(r_{u j}, r_{N(j)}\right)\right]
$$

The motivation behind this criterion is the observation that when a new item is added to an user's list, the energy of the system decreases if the item is compatible with the user. Thus, the lower the energy, the more preferable item. The same argument leads to another criterion - the change in free-energy:

$$
s_{j}^{\prime}=\sum_{r_{u j}} \exp \left(-E\left(r_{u j}, r_{N(j)}\right)\right)
$$

In [14], a MRF based solution has been introduced for ranking, but without sparse MRFs.

- An undesirable effect of the hubness property found in practice is that for some hub users and items, the conditional distribution $P\left(r_{u i} \mid \mathscr{R}_{\neg u i}\right)$ could be peaked due to many contributions from

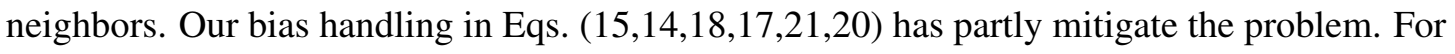
sparsely connected users and items, we can assume that the unseen ratings are the mean rating, thus the pairwise potentials that link with those unseen ratings are close to unity, i.e., $\psi_{i j}, \varphi_{u v} \approx 1$. Alternatively, we could normalize the energy function against the size of the neighborhood size. However, we found that this technique has little effect on the final prediction performance. 
- Finally, it might be useful incorporate social graphs, or item graphs learnt from external sources into our framework.

\section{References}

\section{References}

[1] A. Agresti. Categorical data analysis. Wiley-Interscience, 1990.

[2] K. Ali and W. Van Stam. TiVo: making show recommendations using a distributed collaborative filtering architecture. In Proceedings of the tenth ACM SIGKDD international conference on Knowledge discovery and data mining, pages 394-401. ACM, 2004.

[3] Julian Besag. Spatial interaction and the statistical analysis of lattice systems (with discussions). Journal of the Royal Statistical Society Series B, 36:192-236, 1974.

[4] A.S. Das, M. Datar, A. Garg, and S. Rajaram. Google news personalization: scalable online collaborative filtering. In Proceedings of the 16th international conference on World Wide Web $(W W W)$, pages 271-280. ACM Press New York, NY, USA, 2007.

[5] Aaron Defazio and Tibério S Caetano. A graphical model formulation of collaborative filtering neighbourhood methods with fast maximum entropy training. In Proceedings of the 29th International Conference on Machine Learning (ICML-12), pages 265-272, 2012.

[6] Rosta Farzan and Peter Brusilovsky. Social navigation support in a course recommendation system. In Adaptive hypermedia and adaptive web-based systems, pages 91-100. Springer, 2006.

[7] Asela Gunawardana and Christopher Meek. Tied Boltzmann machines for cold start recommendations. In Proceedings of the 2008 ACM conference on Recommender systems, pages 19-26. ACM, 2008.

[8] J.M. Hammersley and P. Clifford. Markov fields on finite graphs and lattices. Unpublished manuscript, 1971.

[9] D. Heckerman, D.M. Chickering, C. Meek, R. Rounthwaite, and C. Kadie. Dependency networks for inference, collaborative filtering, and data visualization. The Journal of Machine Learning Research, 1:49-75, 2001. 
[10] G.E. Hinton. Training products of experts by minimizing contrastive divergence. Neural Computation, 14:1771-1800, 2002.

[11] T. Hofmann. Latent semantic models for collaborative filtering. ACM Transactions on Information Systems (TOIS), 22(1):89-115, 2004.

[12] Y. Koren. Factor in the neighbors: Scalable and accurate collaborative filtering. ACM Transactions on Knowledge Discovery from Data (TKDD), 4(1):1, 2010.

[13] Greg Linden, Brent Smith, and Jeremy York. Amazon.com recommendations: Item-to-item collaborative filtering. IEEE Internet Computing, 7(1):76-80, 2003.

[14] Shaowu Liu, Gang Li, Truyen Tran, and J Yuan. Preference Relation-based Markov Random Fields. In Proc. of 7th Asian Conference on Machine Learning (ACML), Hongkong, November 2015.

[15] Shaowu Liu, Truyen Tran, Gang Li, and J Yuan. Ordinal random fields for recommender systems. In Proc. of 6th Asian Conference on Machine Learning (ACML), Nha Trang, Vietnam, November 2014.

[16] Jie Lu, Dianshuang Wu, Mingsong Mao, Wei Wang, and Guangquan Zhang. Recommender system application developments: A survey. Decision Support Systems, 74:12-32, 2015.

[17] B. Marlin. Modeling user rating profiles for collaborative filtering. In Advances in Neural Information Processing Systems, volume 16, pages 627-634. MIT Press, Cambridge, MA, 2004.

[18] C Martinez-Cruz, C Porcel, J Bernabé-Moreno, and E Herrera-Viedma. A model to represent users trust in recommender systems using ontologies and fuzzy linguistic modeling. Information Sciences, 311:102-118, 2015.

[19] P. Resnick, N. Iacovou, M. Suchak, P. Bergstorm, and J. Riedl. GroupLens: An open architecture for collaborative filtering of netnews. In Proceedings of ACM Conference on Computer Supported Cooperative Work, pages 175-186, Chapel Hill, North Carolina, 1994. ACM.

[20] R. Salakhutdinov and A. Mnih. Probabilistic matrix factorization. Advances in neural information processing systems, 20:1257-1264, 2008. 
[21] R. Salakhutdinov, A. Mnih, and G. Hinton. Restricted Boltzmann machines for collaborative filtering. In Proceedings of the 24th ICML, pages 791-798, 2007.

[22] B. Sarwar, G. Karypis, J. Konstan, and J. Reidl. Item-based collaborative filtering recommendation algorithms. In Proceedings of the 10th international conference on World Wide Web, pages 285295. ACM Press New York, NY, USA, 2001.

[23] A Tejeda-Lorente, C Porcel, J Bernabé-Moreno, and E Herrera-Viedma. REFORE: A recommender system for researchers based on bibliometrics. Applied Soft Computing, 30:778-791, 2015 .

[24] Nenad Tomašev, Miloš Radovanović, Dunja Mladenić, and Mirjana Ivanović. The role of hubness in clustering high-dimensional data. In Advances in Knowledge Discovery and Data Mining, pages 183-195. Springer, 2011.

[25] T.T. Truyen, D.Q. Phung, and S. Venkatesh. Preference networks: Probabilistic models for recommendation systems. In P. Christen, P.J. Kennedy, J. Li, I. Kolyshkina, and G.J. Williams, editors, The 6th Australasian Data Mining Conference (AusDM), volume 70 of CRPIT, pages 195-202, Gold Coast, Australia, Dec 2007. ACS.

[26] T.T. Truyen, D.Q. Phung, and S. Venkatesh. Ordinal Boltzmann machines for collaborative filtering. In Twenty-Fifth Conference on Uncertainty in Artificial Intelligence (UAI), Montreal, Canada, June 2009.

[27] Jun Wang, Arjen P De Vries, and Marcel JT Reinders. Unifying user-based and item-based collaborative filtering approaches by similarity fusion. In Proceedings of the 29th annual international ACM SIGIR conference on Research and development in information retrieval, pages 501-508. ACM, 2006.

[28] Jun Zou, Arash Einolghozati, Erman Ayday, and Faramarz Fekri. Iterative similarity inference via message passing in factor graphs for collaborative filtering. In Information Theory Workshop (ITW), 2013 IEEE, pages 1-5. IEEE, 2013. 